\title{
Précarités professionnelles et action collective
}

La forme syndicale à l'épreuve

Trade-unions and precariousness. Precarious employment and collective action. A challenge for trade-unions

\section{Paul Bouffartigue}

\section{OpenEdition \\ Journals}

Édition électronique

URL : http://journals.openedition.org/travailemploi/4045

DOI : 10.4000/travailemploi.4045

ISSN : $1775-416 \mathrm{X}$

\section{Éditeur}

DARES - Ministère du Travail

\section{Édition imprimée}

Date de publication : 5 décembre 2008

Pagination : 33-43

ISSN : 0224-4365

Référence électronique

Paul Bouffartigue, "Précarités professionnelles et action collective», Travail et Emploi [En ligne]

116 | octobre-décembre 2008, mis en ligne le 05 novembre 2010, consulté le 30 avril 2019. URL

http://journals.openedition.org/travailemploi/4045; DOI : 10.4000/travailemploi.4045 


\title{
Précarités professionnelles et action collective. La forme syndicale à l'épreuve
}

\author{
Paul Bouffartigue (*)
}

Trois études de terrains, contrastés au plan des formes de précarisation de l'emploi et des modalités de l'action syndicale et collective, montrent que la notion de précarité est relative, polémique et normative et comment elle remet en question les assises sociales et institutionnelles du syndicalisme. À La Poste, le syndicalisme parvient à contenir la précarisation de l'emploi et à renouveler son influence chez les salariés qui intègrent l'entreprise via l'emploi instable. Sur des sites pétrochimiques, quelques expériences, fragiles tentent de faire face à la précarité sous-traitée. Des grèves ont éclaté dans la restauration rapide, sans que le syndicalisme ne s'y implante significativement. La remise en question de la forme syndicale est d'autant plus radicale qu'est bouleversée la pertinence de la double référence à l'entreprise et à la branche d'activité comme fondements de l'organisation et de la représentation syndicale et que l'espace professionnel concerné offre peu de perspectives de stabilisation.

Le lien étroit entre la précarisation de l'emploi et du travail et l'affaiblissement du syndicalisme semble aller de soi. L'étymologie du mot «précaire»-precarius, «obtenu par la prière»-, comme le mouvement de la jeunesse scolarisée contre le «CPE»-exprimant autant le refus de l'arbitraire que de l'incertitude du lendemain - rappellent que la mise en insécurité des travailleurs signifie d'abord leur soumission au pouvoir discrétionnaire des employeurs. Cependant, faute d'interroger l'évidence de ce lien on risque de le naturaliser: les processus qui sont au principe de la crise profonde du mouvement syndical ne débordent-ils pas amplement ceux qui tiennent à la déstabilisation des formes d'emploi et au chômage de masse? Et le retour de l'insécurité sociale n'offre-t-il pas des espaces à l'action collective pour la dignité de la condition salariale, dans un contexte où tout montre la persistance d'une conflictualité sociale?

\footnotetext{
* LEST-CNRS, université Aix-Marseille I et II. E-mail paul. bouffartigue@univmed.fr Une première version de ce texte a été présentée aux «XI ${ }^{\text {es }}$ Journées internationales de sociologie du travail- restructurations, précarisation et valeurs » (Londres 20-21-22 juin 2007) sous le titre: Précarités professionnelles et action collective. Enseignements d'une recherche sur trois terrains contrastés. Il s'appuie sur une recherche réalisée pour le compte de la Dares: P. Bouffartigue, J.-R. Pendariès, F. Peroumal, E. Perrin (Avec la participation de J. Bouteiller, S. Contrepois, B. Fribourg, R. Jean, E. Orban, T. Samzun), Action collective et précarités. le syndicalisme à l'épreuve. Enquête sur trois secteurs d'activité: La Poste, sous-traitance pétrochimique, restauration rapide, LEST, mars 2007. Sur les trois terrains, c'est le cas de la CGT qui a été privilégié, compte tenu de sa prépondérance dans les deux premiers, et de sa plus présence dans les grèves dans le troisième.
}

En posant l'hypothèse qu'il s'agit de la crise d'une forme historique du syndicalisme - celle héritée de la période keyneso-fordienne - , en étudiant sur des terrains d'enquête diversifiés la manière dont les syndicats affrontent les précarités professionnelles et dont s'exprime la conflictualité, on se veut attentif aux signes de renouvellement et de ressourcement pouvant dessiner une nouvelle forme syndicale.

En adoptant une définition large a priori de la précarité professionnelle, on peut explorer d'abord une large palette des formes concrètes de la déstabilisation du salariat, avant de s'interroger sur les relations entre les difficultés du syndicalisme et la déstabilisation de l'emploi. À La Poste, ensuite, le nouveau visage du clivage «statutaires»/《hors statuts» ne laisse pas le syndicalisme désarmé, d'autant plus que ce dernier conserve des forces significatives. Sur les sites pétrochimiques, la précarité apparait largement «sous-traitée» et plus difficile à combattre. Dans la restauration rapide, enfin, le turn-over considérable et le poids des étudiants semblent constituer un obstacle guère surmontable à une implantation syndicale, malgré l'apparition de quelques grèves spectaculaires. Pour finir, la comparaison de ces trois configurations montre l'enjeu crucial des modèles pertinents de sécurisation professionnelle à opposer aux précarités professionnelles.

\section{Définir la précarité}

Plutôt que d'enfermer a priori la notion de précarité dans une définition savante, il est apparu fécond de prendre acte de sa puissance et de sa polysémie dans les représentations ordinaires du 
monde social. Catégorie normative et polémique s'il en est, elle est utilisée d'abord pour dénoncer comme a-normale une situation ou une condition au regard d'une norme positive de stabilité ou de sécurité. Catégorie toujours relative et relationnelle donc, c'est une construction sociale susceptible de connaître de grandes variations selon les contextes et les acteurs. La distance semble considérable entre le CDD de La Poste, inscrit dans une sorte de file d'attente pour l'accès à l'emploi stable de la grande firme publique, et l'équipier de Mc Donald's ne passant que quelques semaines dans un des multiples «petits boulots» nécessaires à la poursuite de ses études. Et pourtant dans chacun des cas la notion d'emploi précaire fait sens.

Toutefois, quelques points de repères conceptuels et empiriques doivent servir de boussole. Avec P. Cingolani (2005) on distinguera trois sens: le travail ou l'emploi précaire, les salariés précaires, la précarité comme manifestation spécifique de la pauvreté, en s'intéressant ici surtout aux deux premiers. On précisera, avec S. Paugam (2002), que emploi et travail précaires ne se confondent pas mais se combinent diversement et se superposent parfois. La notion de précarité d'emploi est liée au contrat de travail - précarité de droit-, elle n'est qu'une composante de l'instabilité d'emploi - précarité de fait; celle de précarité du travail s'applique à l'activité de travail, ses conditions, sa reconnaissance pratique et symbolique. La précarité d'emploi de droit est la plus visible, désignant les formes juridiques qui s'écartent de la norme du CDI à temps plein avec un seul employeur. La précarité du travail ne concerne pas que les salariés en emploi précaire mais les affecte plus systématiquement. On ajoutera à la précarité de l'emploi et du travail la précarité des relations professionnelles - ineffectivité ou faible application du droit du travail et du droit syndical - composantes actives des premières sur deux de nos trois terrains d'enquête.

Tous les salariés sur statut d'emploi précaire ou dans des situations d'emploi précaires de fait ne se vivent pas et/ou ne peuvent être considérés ipso facto comme précaires. La distinction entre les formes d'emploi ou de travail «non traditionnelles», et les caractéristiques sociales des travailleurs, permet d'éviter la pente misérabiliste d'un tel amalgame (Bourhis, Wills, 2001). Maints travaux ont montré que la discontinuité des périodes d'emploi pouvait, dans certaines conditions, être relativement maîtrisée par les salariés.

Reste que les statuts d'emploi précaires sont aujourd'hui dans trois cas sur quatre subis et non choisis, et qu'ils protègent cinq fois moins bien du chômage que les emplois sur CDI. Les jeunes y sont nettement surexposés sans que ces emplois soient réservés à une «file d'attente» dans laquelle patienteraient les «outsiders» aux portes du noyau de stabilité des «insiders». Si une majorité d'entre eux peut espérer quitter le monde du travail précaire et non qualifié en cours de carrière, ce n'est pas nécessairement le cas pour ceux qui cumulent faibles ressources scolaires et position dominée dans les rapports de sexe ou de race (AMOSSÉ, CHARDON, 2006).

\section{Déstabilisation de l'emploi et difficultés du syndicalisme}

Apprécier le rôle qu'a pu jouer la déstabilisation de l'emploi dans la crise du syndicalisme en France excède largement l'ambition de ce texte. On sait combien cette dernière fait l'objet de thèses distinctes. Certains chercheurs mettent en avant son institutionnalisation et sa professionnalisation, associées à son éloignement de sa base sociale (Adolfatto, LabBÉ, 2006). D'autres avancent d'abord le rôle de la crise économique, comme de la division et de la désorientation stratégique des syndicats (Mouriaux, 2005). Cette crise touche la plupart des pays anciennement industrialisés, mais elle est particulièrement accusée en France. Un simple regard sur l'évolution du taux de syndicalisation dans notre pays depuis soixante ans suggère la complexité et la multicausalité du phénomène.

Plus du quart des salariés est syndiqué dans l'immédiat après-guerre, contre $8 \%$ aujourd'hui (Amossé, 2004). Mais cette chute sanctionne une évolution en quatre temps : une diminution rapide et régulière jusque la fin des années 1950; une légère remontée des années 1960 au milieu des années 1970; un nouveau déclin jusqu'au début des années 1990, suivi d'une stabilisation. On le sait, en longue durée, le taux de syndicalisation en France apparaît comme l'un des plus faibles des pays industriels, les périodes d'intense conflictualité sociale et politique - le Front Populaire, la Libération - se traduisant par des vagues de syndicalisation relativement éphémères. Au cours des «Trente Glorieuses» les évolutions apparaissent donc paradoxales : ressac de la vague exceptionnelle de la Libération, puis consolidation associée à celle de la "société salariale» et du compromis économique et social keynesofordien qui singularise cette période. La courbe de la désyndicalisation qui intervient à partir de la fin des années 1970 est associée de manière suffisamment étroite avec celle de la montée du chômage et de la déstabilisation de l'emploi pour admettre qu'il s'agit de l'un des facteurs cette chute.

«Les formes d'emploi flexibles sont de fait un obstacle à la participation syndicale: seuls 2,4\% des salariés en CDD ou en intérim sont syndiqués » (Amossé, Pignoni, 2006). En dehors de la CGT, tous les syndicats ont délaissé l'organisation des chômeurs. Le syndicalisme apparaît aujourd'hui replié dans le secteur public et les grandes firmes du secteur privé, au sein desquels il est souvent davantage représentatif du salariat intermédiaire que des 
travailleurs du bas de l'échelle. Plusieurs recherches ont montré les relations entre précarisation professionnelle et sociale, et prise de distance avec la vie syndicale et politique. Des comparaisons internationales enfin ont montré combien l'emploi précaire mettait partout le syndicalisme au défi de repenser son mode de contribution à l'unification du salariat (Dufour, Hege, 2005).

L'affaiblissement du syndicalisme et le recul en longue durée des journées de grève, indicateur d'une forme longtemps canonique d'action salariale, ne signifie pas la fin de la conflictualité sociale au travail, mais le renouvellement et l'invisibilisation partielle de cette dernière: si sa forme canonique, la grève, est en déclin, d'autres formes - refus d'heures supplémentaires, pétitions, débrayages de courte durée - progressent (DENIS, 2005; Carlier, Tenret, 2007). Apparaissent plus répandues encore les situations d' " esprit de révolte sans révolte» (ZARIFIAN, 2005), de « consentement critique» (MARTUCELli, 2004), ou de résistance individuelle qui fournissent un terreau potentiel de ressourcement de l'action collective.

Le syndicalisme a toujours été confronté au défi de la construction d'une représentation commune du salariat à partir de l'hétérogénéité de ce dernier. $\mathrm{Au}$ début du $\mathrm{XX}^{\mathrm{e}}$ siècle en France, c'est la branche d'activité professionnelle qui est devenue sa base organisationnelle, sur laquelle l'entreprise est, plus tard, venue se greffer. Son influence s'est diffusée de ses places fortes vers les périphéries du salariat au travers du mécanisme de la régulation fordienne. C'est ce processus qui s'est inversé avec la crise de cette dernière. En particulier, la dispersion du salariat dans des établissements de petite taille et la multiplicité des branches professionnelles dont relèvent les travailleurs intervenant sur un même site sont des cas de figure qui interrogent radicalement le mode d'organisation syndical. À quelles conditions et sous quelles modalités le syndicalisme pourrait-il renouer avec une ambition de solidarisation de fragments d'un monde du travail aujourd'hui émietté? Ces questions se posent a priori de manière assez différente selon les contextes qui combinent modalités de la précarité professionnelle et ressources syndicales.

Les trois terrains étudiés correspondent à trois cas de figure typiques des modalités de déstabilisation du salariat, associées à des ressources syndicales différenciées ( $c f$. tableau 1 et encadré 1). La Poste est une grande entreprise publique dans laquelle il s'agit principalement d'une dualisation des statuts d'emploi chez le même employeur, avec un syndicalisme encore bien implanté chez les salariés stables (1). La pétrochimie correspond à de grandes

(1) L'emploi précaire est estimé à $15 \%$ dans la fonction publique, le taux de syndicalisation également (contre $5 \%$ dans le secteur privé).

\section{Encadré 1 \\ Méthodologie}

L'enquête, principalement qualitative, s'est déroulée entre 2003 et 2006, dans la région de Marseille et de l'étang de Berre pour les deux premiers terrains, en région parisienne pour le troisième. Elle est basée principalement sur la réalisation de soixante entretiens auprès de syndicalistes et de salariés non syndiqués.

La Poste. L'étude a été conduite principalement dans ce qui est désormais l'un des quatre «métiers" du groupe, le courrier, plus précisément la distribution (non les centres de tri). Les emplois concernés sont donc ceux de "facteurs" et d' "agents de collecte et de tri » rattachés aux bureaux. L'activité courrier mobilise dans le département étudié 5000 des 9000 emplois offerts par La Poste. Deux monographies de bureaux ont été réalisées: une unité de taille moyenne (37 tournées, 67 emplois), fortement syndiquée, mais au climat social assez consensuel; et une unité de grande taille (250 tournées, 400 emplois), peu syndiquée, récemment réorganisée sur la base de la centralisation et de l'alphabétisation du «tri général », au prix d'un important conflit. Une vingtaine d'entretiens individuels ont été réalisés auprès de responsables de l'entreprise $(n=6)$, de syndicalistes $(n=7)$, de salariés précaires $(n=9)$. Sept autres précaires ont participé à un "groupe de paroles". Elle s'appuie également sur une enquête postale auprès de 450 précaires et CDI récents (155 questionnaires retournés). Sept autres entretiens ont été réalisés auprès de jeunes grévistes de l'activité colis (ColiPoste), sur CDI mais presque tous anciens "précaires".

Sous-traitance pétrochimique. Seize entretiens individuels et collectifs ont été conduits auprès d'une trentaine de syndicalistes, dont des militants de quatre sous-traitants de premier rang (métallurgie-mécanique, et instrumentation-électricité). On s'est focalisé sur deux principaux sites: PETROX (complexe pétrochimique comprenant une raffinerie et plusieurs unités chimiques), employant 1500 salariés directs, et autant de salariés extérieurs. Vingt des 500 entreprises sous-traitantes absorbent $65 \%$ du chiffre d'affaires sous-traité; et CHIMIX, qui fabrique des produits issus du chlore, emploie 380 personnes, et sous-traite avec cinquante firmes. Une enquête par questionnaire a également été réalisée auprès de 100 salariés accueillis à la permanence juridique d'une des UL.

Restauration rapide. Quatorze entretiens ont été réalisés auprès de syndicalistes et d'animateurs des grèves du début des années 2000, principalement chez Mc Donald's (grèves de 2001-2002, puis de 2003,-2004 au restaurant de Strasbourg SaintDenis), secondairement chez Pizza Hut. Ces grèves ont également fait l'objet d'observation directe. Tracts, articles de presse et film documentaires ont également nourri l'analyse de ces conflits.

firmes privées où la précarité d'une fraction des travailleurs intervenant sur les sites est en quelque sorte «sous-traitée » à d'autres employeurs, et où la syndicalisation ne concerne que les salariés orga- 
Tableau 1 : Les trois secteurs d'activité étudiés

\begin{tabular}{|c|c|c|c|}
\hline & La Poste & $\begin{array}{l}\text { Sous-traitance } \\
\text { pétrochimique }\end{array}$ & Restauration rapide \\
\hline $\begin{array}{l}\text { Effectif: } \\
\text { dont «précaires» }\end{array}$ & $\begin{array}{l}300000 \text { (groupe, } 2005 \text { ) } \\
\text { CDD et CDII : } 8 \%\end{array}$ & $\begin{array}{l}15000 \text { (étang de Berre, } 2000 \text { ) } \\
\text { Dont } 20 \text { à } 30 \% \text { en sous-traitance }\end{array}$ & $\begin{array}{l}113000(2004) \\
\text { CDD: } 7 \% \\
\text { Temps partiel: } 64 \% \\
\text { Moins d'un an d'ancienneté: } 34 \%\end{array}$ \\
\hline Précarité de l'emploi & $\begin{array}{l}\text { 1) CDD } \\
\text { 2) Autres: CDI temps partiel } \\
\text { intérim, sous-traitance }\end{array}$ & $\begin{array}{l}\text { 1) Fragilité des sous-traitants } \\
\text { 2) Emplois précaires chez } \\
\text { les entreprises sous-traitantes }\end{array}$ & $\begin{array}{l}\text { 1) Instabilité main-d'œuvre } \\
\text { 2) Temps partiel }\end{array}$ \\
\hline Précarité du travail & $\begin{array}{l}\text { Polyvalence, avec } \\
\text { formation insuffisante } \\
\text { Ex.: facteurs «rouleurs» }\end{array}$ & $\begin{array}{l}\text { Externalisation du «sale boulot»: dont: } \\
\text { enjeu santé au travail } \\
\text { Fragment-précarisation } \\
\text { des collectifs de travail } \\
\text { Nomadisme géographique }\end{array}$ & $\begin{array}{l}\text { Intensité du travail } \\
\text { Horaires de travail flexibles et } \\
\text { fragmentés }\end{array}$ \\
\hline $\begin{array}{l}\text { Précarité des relations } \\
\text { professionnelles }\end{array}$ & $\begin{array}{l}\text { «Abus» dans l'usage CDD } \\
\text { «un CDD ne fait pas grève» } \\
\text { Mais forte syndicalisation chez } \\
\text { salariés stables }\end{array}$ & $\begin{array}{l}\text { Généralisée, sauf chez quelques entre- } \\
\text { prises } \\
\text { sous-traitantes de premier rang }\end{array}$ & $\begin{array}{l}\text { Généralisée sauf implantation } \\
\text { Syndicale ponctuelle }\end{array}$ \\
\hline $\begin{array}{l}\text { Profil social } \\
\text { de la main-d'œuvre } \\
\text { précaire }\end{array}$ & $\begin{array}{l}\text { CDD: jeunes, mixité } \\
\text { proches des postiers stables }\end{array}$ & $\begin{array}{l}\text { Ouvrier, masculin } \\
\text { Qualifié (dont «mercenaires») } \\
\text { Et non qualifié }\end{array}$ & $\begin{array}{l}\text { Jeunes, mixité } \\
\text { Étudiants: origine populaire } \\
\text { Svt. immigrée. Très instables } \\
\text { Autres salaires. Moins instables }\end{array}$ \\
\hline $\begin{array}{l}\text { Avenir professionnel } \\
\text { pensable pour } \\
\text { les salariés }\end{array}$ & $\begin{array}{l}\text { Emploi stable à La Poste } \\
\text { pour une partie importante }\end{array}$ & $\begin{array}{l}\text { «Tenir» pour les «mercenaires », stabi- } \\
\text { lité de l'emploi et territoriale } \\
\text { pour les autres }\end{array}$ & $\begin{array}{l}\text { Quitter le restaurant et le secteur } \\
\text { (majorité) } \\
\text { Promotion hiérarchique (minorité) }\end{array}$ \\
\hline $\begin{array}{l}\text { Mobilisation des } \\
\text { précaires }\end{array}$ & Non & Non & Oui \\
\hline $\begin{array}{l}\text { Stratégie collective } \\
\text { de sécurisation } \\
\text { professionnelle }\end{array}$ & $\begin{array}{l}\text { Respect du Code du travail } \\
\text { Limiter l'emploi précaire } \\
\text { Transformation CDD et CDI } \\
\text { à temps partiel en CDI temps } \\
\text { complet }\end{array}$ & $\begin{array}{l}\text { Respect du Code du travail } \\
\text { Homogénéiser conditions HSCT } \\
\text { Limiter l'emploi précaire } \\
\text { Reprise acquis en cas licenciement }\end{array}$ & $\begin{array}{l}\text { Respect du Code du travail } \\
\text { Reconnaissance de l'ancienneté }\end{array}$ \\
\hline $\begin{array}{l}\text { Obstacles spécifiques } \\
\text { à l'action syndicale }\end{array}$ & $\begin{array}{l}\text { Télescopage syndicalisme } \\
\text { fonctionnaire/salariat privé }\end{array}$ & $\begin{array}{l}\text { Division intra-salariale, organiques/ } \\
\text { sous-traitants } \\
\text { Répression }\end{array}$ & $\begin{array}{l}\text { Instabilité, rapport } \\
\text { Instr. au travail (étudiants) } \\
\text { dispersion, soumission } \\
\text { Répression. Turn-over } \text { syndical }\end{array}$ \\
\hline Défis posés & $\begin{array}{l}\text { Organiser les précaires } \\
\text { Contenir la flexibilité interne } \\
\text { Crise du syndicalisme } \\
\text { Peser sur avenir de La Poste }\end{array}$ & $\begin{array}{l}\text { Organiser les entreprises ST } \\
\text { Sortir de l'entreprise, du site et de la } \\
\text { branche } \\
\text { Reconstruire entité pertinente } \\
\text { Articuler aide individuelle } \\
\text { et mobilisation collective } \\
\text { Crise du syndicalisme } \\
\text { Peser/avenir branche et firmes }\end{array}$ & $\begin{array}{l}\text { Organiser les salariés } \\
\text { Sortir de l'entreprise } \\
\text { Alliance clients/autres mouvements } \\
\text { Articuler aide individuelle } \\
\text { et mobilisation collective } \\
\text { Crise du syndicalisme }\end{array}$ \\
\hline
\end{tabular}

niques et quelques rares grands sous-traitants de «premier rang». La restauration rapide enfin forme un secteur qui est dans son ensemble marqué par une très forte instabilité de la main-d'œuvre, une très faible présence syndicale, mais par l'apparition ces dernières années de quelques grèves parfois très longues. Nous avons donc trois configurations très contrastées au plan de l'histoire du secteur d'activité, du statut de l'employeur principal, de la présence syndicale, comme du profil social des salariés. Mais dans les trois cas se superposent étroitement, sans bien sûr se recouvrir, instabilité d'une fraction plus ou moins importante des salariés, et difficultés de la syndicalisation. Sur ces trois terrains enfin le syndicalisme concerné est incarné par la CGT, soit qu'il s'agisse du seul syndicat animateur des conflits étudiés (restauration rapide) ou initiateur d'expériences de coordinations de site ou inter-sites (sous-traitance pétrochimique), soit qu'il soit en position localement très dominante (La Poste). On ne prétend donc pas rendre compte ici de la totalité des orientations et pratiques syndicales eu égard à la flexibilité de l'emploi.

\section{La Poste: la précarité de l'emploi contenue}

Deux traits majeurs semblent distinguer la «plus grande entreprise» de France: la résistance de l'emploi statutaire; la puissance des syndicats, et l'orientation contestataire des deux plus influents (CGT et SUD). Mais une révolution silencieuse s'opère au sein de l'emploi statutaire, avec la montée en puissance d'un salariat de droit privé, 
suite à la transformation de La Poste d'une administration d'État à une entreprise publique: aux côtés des fonctionnaires, les agents contractuels - «Acos»-représentent aujourd'hui $40 \%$ des effectifs.

Si la part des emplois de statut précaire au sens strict - CDD, intérim, CDII (contrat à durée indéterminée intermittent)-oscille de 8 à $11 \%$ dans l'ensemble des effectifs au cours des dix dernières années, il faudrait leur ajouter les CDI à temps partiel contraint, également considérés comme "précaires» dans les derniers accords collectifs de «déprécarisation». Le fort taux de syndicalisation (20 à $25 \%$ ), ne doit pas occulter l'affaiblissement et la division des syndicats, ou la non généralisation de conflits du travail, nombreux mais localisés. Enfin les «Acos» ont un statut professionnel sensiblement dégradé eu égard aux fonctionnaires, et ce sont pour la plupart d'anciens CDD qui forment ainsi un vecteur central du remodelage en cours de la main-d'œuvre(2). Les hésitations des orientations syndicales à leur endroit sont significatives du paradoxe face auquel elles se trouvent: devoir s'emparer de la défense des salariés appelés à devenir majoritaires, sans renoncer à se référer au fonctionnariat comme modèle statutaire. C'est au travers de tensions internes et après un certain délai d'adaptation que les syndicats se sont tournés vers les «Acos». Mais d'un syndicaliste à l'autre, la notion de précarité prend des contours très variables: très larges, quand elle désigne l'ensemble de ceux qui ne sont pas fonctionnaires; plus étroits, quand elle se limite aux seuls contractuels écartés du CDI à temps plein.

Dans le secteur de la distribution du courrier, les CDD sont utilisés principalement à la fois pour remplacer des absents - notamment lors des congés , occuper des postes laissés vacants du fait d'un sous-effectif chronique ou de la longueur des délais d'affectation, et former et sélectionner les agents, dont certains sont destinés à être recrutés en CDI. Deux profils polaires y cohabitent, entre lesquels les frontières ne sont pas étanches: des salariés en quête d'un salaire d'appoint ou d'un emploi d'attente, tels des étudiants, et d'autres, s'inscrivant dans une voie d'accès à l'emploi postal stable.

La précarité du statut se redouble très nettement d'une précarité du travail: tous les facteurs en CDD «roulent» sur plusieurs tournées, chacune nécessite un temps d'apprentissage au cours duquel la durée de distribution dépasse celle du facteur «titulaire»

(2) Leur salaire est estimé inférieur d'un tiers à celui des fonctionnaires (ZARIFIAN, 2003). de sa tournée (3). Ces séquences d'apprentissage supposent un fort engagement professionnel, sans autre contrepartie que l'espérance d'une stabilisation, ou, à l'inverse, la contrepartie de la brièveté de la durée d'emploi. Si cette polyvalence reste généralement limitée au cadre d'un même bureau et du même type d'emploi, elle peut s'étendre à plusieurs établissements et types d'emploi.

La précarité des relations professionnelles, bien moins marquée que sur nos deux autres terrains, n'est cependant pas inconnue à La Poste : la fréquence des recours juridiques pour «usage abusif» de CDD le montre, des responsables des ressources humaines de l'entreprise reconnaissant volontiers leurs difficulté d'appropriation du Code du travail et de la complexité des règles juridiques dans un monde professionnel hybridant les références à la fonction publique et au droit privé.

$\mathrm{Au}$ cours des dernières années, la précarité de l'emploi a été à la fois un des thèmes centraux explicites de l'activité syndicale, sous la forme de recours juridiques - particulièrement prisés par SUD - , de la négociation collective et de l'activité syndicale ordinaire, et composante récurrente des conflits portant d'abord sur les conditions de travail et sur les effectifs (4).

À la fin des années 1990, à la veille des négociations sur les «35 heures», $40 \%$ des «Acos» sont sur CDD ou CDII. Les recrutements réalisés à cette occasion sont, pour une part, des transformations d'emplois précaires en emplois stables. En 2004 sont signés dans l'activité courrier par tous les syndicats, sauf SUD, les «accords de Vaugirard». Leur mesure phare est la «fin du temps partiel imposé» et la transformation de 10000 CDD en CDI sur deux années (5). Deux années plus tard le bilan apparaît mitigé. L'emploi précaire se tasse, mais les recrute-

(3) Ce qui ne signifie pas que les facteurs bénéficiant d'un CDI échappent tous à cette précarité du travail, notamment au cours de leur période d'apprentissage s'ils sont recrutés directement sur CDI, ou même au-delà de cette période, en attente d'être «titulaire» d'une tournée. Les syndicalistes insistent d'ailleurs sur ce phénomène pour construire du commun entre expériences diversifiées de la précarité celle des CDD et des autres postiers, quitte à parler de «précarisation» de l'ensemble des postiers. Les déclinaisons locales de l'accord de «déprécarisation» dit de Vaugirard ( $c f$. plus loin) prévoient d'ailleurs des contre parties en termes de flexibilité interne, sous forme de polyvalence sur différentes tournées.

(4) Un exemple parmi d'autres. Une grève de trois semaines paralyse en 2006 les trois agences locales de ColiPoste (activité colis du groupe). Le conflit est déclenché par des sanctions liées à des accusations de vol. Il porte également sur le «management par le stress», la charge de travail excessive, mais aussi sur l'emploi: volant de remplacement, brigade de tri, et «passage de tous les CDD en CDI».

(5) La CGT motive sa signature notamment par le fait que l'accord soit réalisé «à partir des revendications des salariés» et non du «cahier revendicatif patronal». SUD justifie son refus par son caractère «insuffisant et incomplet» et son inscription dans «l'éclatement de l'entreprise» (il ne concerne qu'un des quatre métiers du groupe). 
ments sur CDD se poursuivent. L'objectif affiché de leur limitation à $5 \%$ des effectifs s'éloigne.

Dans le département où l'enquête a été conduite, un important conflit intervient début 2005(6) et le dialogue social est pratiquement suspendu pendant plusieurs mois. L'accord national n'est donc décliné que dix-huit mois plus tard. La CGT, ici en position très dominante(7), s'est efforcée d'encadrer les contreparties, qui sont autant de concessions en matière de "principes »-acceptation de «tests» dans la période qui suit la première embauche et non pas avant, et de la polyvalence sous la forme d'une intégration au sein de volants multisites -, contreparties jugées acceptables en échange d'un taux de dé-précarisation supérieur à la moyenne nationale. L'objectif est «que les gens qui rentrent dans la boîte aient une perspective de CDI le plus rapidement possible». De fait, l'accord départemental institutionnalise un parcours de stabilisation, basé sur l'ancienneté et scandé par des seuils, dont le terme est pour les facteurs, au-delà de l'accès au CDI, la «titularisation» sur une seule tournée. La direction de l'entreprise régule ainsi l'embauche et la gestion des débutants dans une perspective plus propice à leur engagement professionnel et au souci de «qualité de service». La question reste posée du poids qu'occupera à l'avenir le recrutement sur CDD par rapport à d'autres modalités : s'il devait rester prépondérant, on pourrait interpréter ce type d'accord comme une régulation conjointe d'une «file d'attente» aux portes d'un marché interne du travail; s'il devait diminuer sensiblement, les syndicats seraient parvenus à réduire une modalité centrale de la précarité professionnelle à La Poste.

Précarité et précaires occupent également une certaine place dans l'activité syndicale ordinaire, nécessairement inégale selon la densité et le dynamisme des équipes syndicales au niveau des établissements: prises de parole, tracts, prise en charge de cas individuels. Toutefois l'interpellation collective des précaires est rare, et la mobilisation collective de ces derniers plus encore. La convention selon laquelle «un CDD ne fait pas grève» est très largement partagée, et leur syndicalisation n'est guère sollicitée. Par contre, en se syndiquant assez massivement peu de temps après l'obtention de leur $\mathrm{CDI}$, les anciens précaires témoignent d'une sorte de reconnaissance de dette à l'égard des syndicats.

(6) Les facteurs se sont mis en grève pendant trois semaines suite à une importante restructuration des activités de distribution sur la seconde agglomération urbaine du département. Le passage au "tri alphabétique» se traduit par l'exclusion des facteurs des opérations de «tri général» et par vingt-cinq suppressions d'emploi. Le travail reprendra avec la sauvegarde de la moitié d'entre eux.

(7) Elle y recueille $51,5 \%$ des voix $(34,8 \%$ au plan national); et $56,5 \%(39,2 \%)$ dans les seules activités courrier. SUD, avec $20 \%$ des suffrages, a localement la même influence qu'au plan national.
Un des ressorts de ce mouvement d'adhésion réside dans la proximité sociale très forte de ces précaires par rapport au milieu postal, plus de la moitié ayant un membre de leur famille travaillant ou ayant travaillé à La Poste (8).

L'action syndicale sur le terrain de la précarité apparaît donc ici relativement efficace, et ce sur un triple plan: elle parvient à contenir la précarisation des statuts d'emploi, à accompagner la stabilisation professionnelle d'une fraction significative des $\mathrm{CDD}$, et, du moins dans le département étudié, à renouveler l'influence syndicale chez les nouvelles générations de postiers. En puisant dans l'héritage des succès des luttes anciennes pour la «titularisation des auxiliaires» le syndicalisme parvient dans ce cas à formuler des réponses encore pertinentes. Reste qu'une partie importante des précaires ne parvient pas à s'inscrire dans la file d'attente, qu'elle est très démunie au cours des séquences de non emploi(9), et que la relation entre syndicats et salariés demeure marquée par l'utilitarisme. Et faute de peser sur les orientations stratégiques d'une entreprise sujette aux logiques de la déréglementation, les syndicats influencent finalement peu les grands choix en matière de gestion de l'emploi et du travail.

\section{Sites pétrochimiques: fragilité des initiatives syndicales face à la "précarité sous-traitée"}

Si le syndicalisme des postiers a mis quelques années à prendre la mesure de la rupture introduite par des «Acos» aux côtés des fonctionnaires, c'est près d'une vingtaine d'années qui s'écoulent entre les années 1970, quand les firmes s'engagent dans des politiques d'externalisation systématique, et le milieu des années 1990, où des syndicats tentent quelques adaptations substantielles de leurs modes de réponse. Ils le font dans un contexte marqué par l'irréversibilité de fait d'une grande partie du phénomène de sous-traitance - la ré-internalisation des activités n'est plus guère crédible - et par leur affaiblissement substantiel au sein même des firmes

(8) Une enquête par questionnaire conduite auprès de 430 CDD et CDI récents (taux de réponse : $40 \%$ ) fait état de $38 \%$ de syndiqués chez les CDI, $5 \%$ chez les CDD. Au cours de notre enquête, une grève a paralysé pendant trois semaines les trois agences locales de ColiPoste. Presque tous ses jeunes leaders sont d'anciens précaires ( $c f$. note 4$)$.

(9) Le vécu difficile des périodes de chômage entre deux CDD fait partie des apports du "groupe de paroles» de précaires de La Poste que nous avons constitué: complexité des démarches et des délais nécessaires pour faire valoir ses droits à indemnisation; statut de «captivité professionnelle» par rapport à cet employeur (accepter un autre emploi c'est prendre le risque de ne plus être disponible pour La Poste, et donc de sortir de «la file d'attente»). "Abandonnés » par l'entreprise au cours de ces moments, ils le sont aussi par les syndicats. 
donneur d'ordre: l'emploi y a été considérablement réduit sans conflits sociaux majeurs via des plans sociaux particulièrement généreux et le développement de l'actionnariat salarié.

Ici la précarité d'emploi ne prend pas d'abord la forme de la précarité des statuts d'emploi, mais de la dépendance et de la fragilité économiques des entreprises, qui s'accentuent tendanciellement au fil d'une "sous-traitance en cascade», accompagnées d'une dégradation de la condition salariale. Emploi, conditions de travail, risques professionnels (10) et relations professionnelles tendent à se détériorer avec le rang de l'employeur formel dans la chaîne des rapports de sous-traitance. À tel point que pour un syndicaliste, le "véritable précaire» est «celui qui ne sait pas ce qu'il devient à plus d'un mois». Ce n'est pas un hasard si aucune implantation syndicale n'a pu être observée au-delà d'entreprises soustraitantes de premier rang.

Il est vrai que les grandes firmes donneur d'ordre ont fait le choix au cours des dernières années de fidéliser une partie de leurs sous-traitants de premier rang, comme les entreprises générales de maintenance. Le salariat de la sous-traitance apparaît donc de plus en plus différencié. Mais il n'est jamais à l'abri de la perte du marché confié à l'employeur, de la non application de l' «obligation de reclassement », ou d'une ré-embauche par le repreneur à des conditions d'emploi inférieures. La précarité des statuts d'emploi est d'autant plus fréquente que l'on s'éloigne du premier cercle des entreprises soustraitantes. La précarité du travail prend la forme de la segmentation des collectifs, de l'isolement et du nomadisme professionnel des ouvriers, de leur surexposition très nette aux accidents du travail et aux maladies professionnelles. Quant à la précarité des relations professionnelles, elle se signale par la fréquence de la répression antisyndicale.

Les syndicalistes rencontrés distinguent volontiers deux types d'ouvriers de la sous-traitance ( $c f$. encadré 2). Le premier, minoritaire, particulièrement «individualiste» et éloigné du syndicalisme, est le «mercenaire». Souvent intérimaire ou faux artisan, son mode d'emploi est choisi et non subi, il s'appuie sur une qualification recherchée pour vendre au meilleur prix une prestation de travail dont les conditions lui importent peu. Le second est celui qui subi sa précarité. C'est d'abord la peur du chômage et l'espoir d'un statut plus stable qui le tiennent à distance du syndicalisme, même s'il peut s'en rapprocher ponctuellement sur un mode instrumental.

Étudiée à partir de la seule CGT, l'activité syndicale en direction de la précarité sous-traitée se déve-

(10) Accidents avec arrêt et occurrence des maladies professionnelles sont sept à dix fois plus élevés que chez les salariés directs du donneur d'ordre.

\section{Encadré 2}

\section{Précarité choisie, précarité subie: les précaires vus par deux syndicalistes}

«ll y a deux sortes d'intérimaires: ceux qui le subissent pour différentes raisons (trop jeunes, trop vieux...), et ceux qui le revendiquent, qui ne veulent que ça, qui ne veulent pas de relation avec les CDI, ceux qu'on appelle les "forfaitaires" - en fait des "indépendants" - "les mercenaires", ceux qui sont payés à la tâche, "au pouce", c'est-à-dire à la longueur de tuyaux soudés et qui se font 1200-1500 francs par jour. C'est énorme ce qu'ils gagnent, c'est quatre fois ce que je gagne. Ce sont des gens qui sont très forts dans leur métier. Ceux-là, c'est même pas la peine d'aller les voir [...] des jeunes autour de 30-35 ans, qui viennent de se marier, "faut que ça rentre" [...]. II faut les voir dans les ateliers, ils ne lèvent pas la tête, parce qu'ils sont payés "au pouce", des gens qui sont très compétents dans leur métier et qui restent 12 heures par jour [...]. Ils sont capables de souder dans des positions inimaginables et c'est toujours nickel. C'est des artistes [...]. Ils ont une force de travail qui est quand même impressionnante parce qu'ils sont attirés par le gain [...]. Dés fois, ils ne mangent pas, ils font non-stop. [...] Je pourrais faire respecter les temps de pause. Mais avec les mercenaires, si je lui dit d'arrêter 20 minutes, je lui fait péter 30 ou 50 euros. II va me dire oui, mais dès que je tourne le dos, il repart». (Syndicaliste, sous-traitant de premier rang).

"Ceux qui subissent la précarité, c'est la peur, et l'espérance d'être bien vu du patron. Les gens ont dans la tête "si je suis CGT, si je me fais voir, si je m'engage je ne travaille plus". On me l'a sorti souvent, "je suis d'accord avec vous, avec ce que vous dites, mais non ...". Ce qui est faux. Parce que dans ma boîte personne ne sait qui est syndiqué, nous on le sait, mais pas le patron [...]. Certains des CDD qui viennent nous voir nous disent qu'ils sont syndiqués isolés, mais personne n'est venu nous voir pour se syndiquer. Ils viennent nous demander de l'aide [...]. Les gars qui viennent te voir, c'est ceux qui pensaient qu'ils allaient être embauchés et qui comprennent qu'ils ne le seront pas. Pour les précaires, le syndicat sert d'avocat, d'assistante sociale, de conseiller juridique, de conseil technique sur les conventions collectives, mais on est pas un outil revendicatif, on est un défenseur ». (Syndicaliste, sous-traitant de premier rang).

loppe selon quatre registres: deux assez classiques, les deux suivants plus novateurs:

- les trois unions locales dont relèvent les sites pétrochimiques accueillent des salariés «isolés» - privés du soutien d'un syndicat d'entreprise -, très souvent précaires, et s'efforcent de les organiser, voire de les soutenir juridiquement(11).

(11) La moitié des personnes accueillies par une des permanences juridiques d'une des UL ne sont pas sur CDI à temps plein. Femmes et jeunes sont également surreprésentés. 
L'implantation au sein des PME, en particulier au sein de sous-traitants reste l'horizon privilégié de cette activité, avec quelques résultats chez des soustraitants de premier rang;

- quand ils sont parvenus à s'implanter au sein d'entreprises sous-traitantes, les syndicats s'efforcent d'y limiter le nombre d'emplois précaires et d'y obtenir des transformations en CDI, d'y faire respecter le droit du travail, ainsi que les normes en matière de conditions de travail, d'hygiène, et de sécurité individuelle et collective.

Conscients que les sources de la précarité se situent très largement en dehors des limites de leur entreprise, ces syndicalistes sont parmi les plus acquis à l'idée selon laquelle il convient de sortir de ces limites. Les risques professionnels sont le domaine privilégié à partir duquel les clauses des contrats de sous-traitance peuvent être dénoncées et modifiées : la surexposition des travailleurs en soustraitance est manifeste, et les enjeux de santé et de sécurité au travail sont très liées dans ces industries aux enjeux de sécurité des installations et de l'environnement.

$-\mathrm{Au}$ travers du «syndicalisme de site» on rencontre des initiatives qui peuvent être prises par des syndicalistes qui relèvent du personnel direct du donneur d'ordre. Ces démarches vont de la défense des salariés de la sous-traitance présents sur le site à la mise en place de collectifs de site (12) en passant par l'action en amont des clauses du contrat de sous-traitance et l'ouverture du CHSCT du donneur d'ordre aux salariés sous-traitants.

- Le dernier modèle d'action transcende plusieurs sites industriels pour s'établir à l'échelon d'une zone territoriale plus large. La pertinence de cette dernière renvoie à l'unité géographique réelle au sein de laquelle circulent nombre de travailleurs de la sous-traitance. C'est dans ce modèle que s'inscrit un collectif départemental multiprofessionnel de la sous-traitance (13), ou une coopération entre une dizaine de CHSCT d'entreprises donneur d'ordre et sous-traitantes de la zone concernée.

Il reste que ces deux derniers types d'initiative restent très fragiles, voire marginaux, et demeurent finalisés de manière «réaliste» par les deux objectifs de base traditionnels: faire appliquer le droit du travail, et implanter le syndicat là où c'est possible.

(12) Un collectif informel existe pendant quelques années à l'UL de la zone, commune dont le site de la Shell est le premier pôle d'activité. Il regroupe des syndicalistes du plus important syndicat de la firme pharmaceutique - ils en sont à l'initiative, à partir de leur expérience sur les questions de santé et de sécurité - et quelques syndicalistes d'entreprises sous-traitantes de rang 1 et de l'intérim.

(13) Plus officiel que le précédent, il est créé en 1997 dans le cadre de l'UD, à l'initiative d'un responsable de la pétrochimie et appuyé par des militants de la construction. Il regroupe trois fédérations professionnelles (chimie, construction et métallurgie), trois UL, et une dizaine d'entreprises.
Pour nombre de syndicalistes, les précaires de la sous-traitance restent invisibles. Les militants les plus impliqués dans leur défense ont d'ailleurs acquis une sensibilité spécifique à la précarité au travers de leur biographie. Les concurrences et hiérarchies au sein du salariat se retrouvent au sein du syndicalisme, mis en demeure de les transgresser en vue de reconstruire des solidarités plus larges en son sein. Définir les nouvelles entités pertinentes de l'action collective apparaît à la fois indispensable, et risqué au regard des héritages qui font encore référence.

\section{Restauration rapide: la portée de grèves improbables}

Dans ce secteur, tout ou presque semble rendre fort improbable l'action collective et l'implantation syndicale classiques(14). S'il symbolise bien le précariat des services, c'est moins au travers des statuts d'emploi offerts - bien qu'il s'agisse massivement d'emploi à temps partiel - que d'une très forte instabilité professionnelle de fait (Nkouachet, 2005).

Pour la plupart des jeunes des deux sexes qui $\mathrm{y}$ transitent - notamment, mais pas seulement, les étudiants - , il s'agit d'un «petit boulot» alimentaire et provisoire parmi d'autres. Au terme de quelques mois on y est déjà «ancien». Loin d'être un obstacle, cette très forte mobilité est l'un des ressorts du consentement à un travail très intense et contraignant, aux côtés des caractéristiques sociales des salariés - proximité des origines et de génération, favorisant une ambiance «familiale» et un rapport ludique aux défis productifs - et de l'organisation du travail, dans laquelle contraintes industrielles, marchandes et domestiques s'épaulent (CARTRON, 2005). Et quand apparaissent des velléités de résistance collective ou syndicale, elles sont vivement combattues par les directions.

Pourtant, plusieurs grèves, dont certaines très longues, sont intervenues au début des années 2000 chez Mc Donald's ou Pizza Hut. Au Mc Donald's de Strasbourg-Saint-Denis à Paris un premier conflit a paralysé le restaurant pendant 115 jours fin 2001, suivi par un second plus long encore - une année - en 2003-2004. Le premier fait suite au changement de gérant,(15) il est déclenché par le licenciement de cinq délégués du personnel "pour vol». Tous sont swing managers-premier niveau d'encadrement - ou managers, et membres de la CGT. La grève, fortement médiatisée et soutenue par un «comité de soutien» extérieur très actif prend fin avec la réinté-

(14) On prend appui ici principalement sur Peroumal F. et PERRIN E., «Luttes et syndicalisme dans la restauration rapide parisienne», in Bouffartigue P. et al., op. cit.

(15) Plus de $80 \%$ des restaurants de cette enseigne relèvent de ce mode de gestion. 
gration des licenciés. Le second est aussi provoqué par le licenciement d'un délégué, membre cette fois de la CGC et par ailleurs directeur adjoint. Il prend cette fois la forme d'une occupation des locaux, le rôle du comité de soutien est plus faible. La CGT n'y participe plus et contrôle de plus près le conflit. Si le délégué licencié est réintégré et une augmentation salariale obtenue, les syndicalistes visés par le licenciement lors du premier conflit acceptent de négocier leur départ. L'implantation syndicale en sort affaiblie, d'autant plus que plusieurs animateurs de la première grève ont pris leur distance avec le syndicat. Trois ans plus tard, dans le même restaurant, c'est une liste «indépendante» qui devance la CGT aux élections professionnelles, laquelle ne conserve que huit adhérents sur une cinquantaine de salariés.

Le rôle déterminant joué dans certains de ces conflits par un «comité de soutien» non strictement syndical et extérieur aux collectifs en grève - ici, au cours de la première grève - a été souligné. Issu du réseau «stop précarité» récemment créé, regroupant des militants venus d'horizons politiques et syndicaux divers, valorisant le pragmatisme et la non instrumentalisation du mouvement, il participe de la construction de la cause gréviste et de la montée en généralité des registres de justification. On passe d'une grève «d'indignation» et de dénonciation personnalisée du gérant à une plate forme revendicative standard incluant des demandes spécifiques au conditions locales de travail, bref à une grève «critique» dans laquelle l'organisation du travail et de l'emploi considérée hier comme «normale» est désormais jugée «anormale» (CARTRON, 2005). D'autres aspects des modalités de la mobilisation collective sont notables: occupation de restaurants non grévistes et diffusion partielle du conflit au sein de la chaîne, usage d'internet et des téléphones portables afin de coordonner quotidiennement les actions, disqualification symbolique de l'enseigne auprès des clients par détournement de ses slogans publicitaires, mise en place d'un éphémère «collectif restauration rapide» au sein de la CGT, qui participera de la construction ultérieure du réseau «stop précarité». Nombre de ces modalités de lutte et des styles militants qui les portent entrent en tension avec la culture portée par les syndicalistes plus institutionnels, tels les permanents, ce qui éclaire en partie la prise de distance opérée ultérieurement par plusieurs des leaders de ces grève par rapport à l'engagement syndical.

Les trajectoires biographiques des jeunes animateurs des grèves montrent que le déclencheur de leur engagement dans l'action est moins la révolte contre les conditions de travail et d'emploi en elles-mêmes, qu'une rupture entre leurs espérances et la réalité de leurs positions professionnelles. Les plus engagés dans l'action sont parmi les plus anciens, par ailleurs souvent membres de la hiérarchie intermédiaire des restaurants. Ils vivent comme une trahison le sort qui leur est fait. Nombreux sont ceux qui, bien que largement issus des immigrations africaines, sont en position de déclassement social et/ou scolaire relatif. Dans tous les cas ils puisent dans des valeurs héritées de leurs univers de socialisation des raisons de refuser d'aller plus loin dans le consentement à des modes de management qui tendent à les mettre en contradiction avec eux-mêmes ou avec leurs collègues «équipiers ». Un engagement syndical durable peut alors - quand il n'est pas «négocié» contre un démission - , devenir un mode de professionnalisation alternatif et un moyen de «tenir» de manière exceptionnellement longue dans un contexte de travail pénible.

Les conflits de la restauration rapide sont-ils destinés à rester sans lendemain? Si l'organisation socio-économique du secteur semble interdire structurellement un mode traditionnel d'organisation syndicale, quelques noyaux de syndiqués parviennent à s'y implanter ici ou là, et des formes de coordination ou de collectif plus larges sont apparues. Au-delà, des expériences militantes sont susceptibles de se diffuser dans les divers mondes du travail précaire desquels nombre de ces salariés risquent de demeurer captifs.

\section{Quels modèles de stabilisation?}

La précarisation professionnelle prend de multiples formes, lesquelles ne se télescopent pas selon la même intensité avec la forme syndicale. Elle semble saper d'autant plus radicalement les fondements du syndicalisme hérité de la période fordienne qu'elle prend des formes elles-mêmes radicales. C'est le cas notamment quand elles conduisent à vider d'une grande partie son contenu la double référence à l'entreprise - unité de temps, de lieu et d'action - et à la branche, comme lieux de stabilité, d'identification et de négociation collectives, bases sur lesquelles cette forme syndicale s'est édifiée. Et quand apparaissent ces vastes espaces de transition ou de relégation professionnelles, qui n'offrent guère de pôles positifs d'identification et de stabilisation.

C'est la conclusion sur laquelle débouche notre comparaison entre trois situations typées à la fois du point de vue des modalités de déstabilisation du salariat et des ressources disponibles en matière d'action syndicale et collective. La seule des trois dans laquelle des résultats significatifs sont obtenus en matière de déprécarisation et de renouvellement de la syndicalisation est celle de la grande entreprise à statut, avec unicité d'employeur pour les stables comme pour les précaires, et où le syndicalisme conserve à la fois des assises institutionnelles et une base sociale relativement fortes. Les quelques initiatives syndicales intervenues dans le monde de la sous-traitance pétrochimique apparaissent d'échelle très modeste eu égard à la puissance du processus d'externalisation des activités et d'éclatement juridique des collectifs productifs. Quant aux unités de la restauration rapide, basée structurellement sur 
l'appel à une main-d'œuvre juvénile hyper mobile, on voit mal comment le syndicalisme pourrait durablement s'y implanter. C'est sans doute ce dernier cas de figure qui invite le plus à renouveler ou à élargir les premières approches «dualistes» des enjeux de la déstabilisation des formes d'emploi (16): désormais cette dernière n'affecte pas que la «périphérie» d'un «noyau» stable, mais caractérise le fonctionnement de secteurs d'activité entiers, notamment dans le commerce et les services.

Pourtant, même dans ces contextes existent des embryons d'action collective et syndicale. Ils reposent toujours sur les moins précaires des précaires, car il n'est pas d'action collective sans un minimum de ressources pour l'action. Ces dernières s'appuient toujours sur des éléments de stabilité des collectifs de travail, présents au cœur-même des zones de précarité, pour des raisons d'exigences productives, d'efficacité, de sécurité ou de qualité des prestations. Si l'emploi divise, le travail unit (BARNIER, 2006) - ou, du moins, divise moins. Certes, une «communauté professionnelle» autonome reste la «communauté pertinente de l'action collective» la plus solide (Segrestin, 1979). La conflictualité vivace à La Poste repose d'abord sur cette dernière. Mais les grèves dans la restauration rapide montrent également l'existence de «communautés-groupe», sans véritable qualification, même si aucune expérience professionnelle n'est dépourvue de dynamique d'apprentissages de capacités nouvelles, et d'attente de reconnaissance de celles-ci. Dans l'expérience de la précarité professionnelle, disqualification, arbitraire et non reconnaissance se heurtent à des résistances, particulièrement chez les nouvelles générations longuement scolarisées. «Après un conflit, même court, les relations sont plus humaines [...] [Les salariés] veulent juste être respectés» (MABROUKI, 2004, p. 32).

Mais il y a loin de ces révoltes à l'organisation collective et à la syndicalisation durables. La question de la forme syndicale qui peut faire sens dans les perspectives individuelles de stabilisation ouvertes aux précaires se pose de manière nécessairement diversifiée selon les contextes. Et la rencontre estelle toujours possible entre précaires et syndicalistes compte tenu de la distance entre les profils sociaux et culturels des uns et des autres? Notre recherche montre qu'elle est incontestablement plus facile quand ces profils sont proches, comme dans le cas de La Poste, que quand ils sont éloignés, comme dans le cas de la restauration rapide. On rejoint ici les conclusions de la comparaison internationale conduite par l'IRES: «L'exigence de renouvellement présentée aux syndicalismes n'est pas nouvelle, mais les transformations liées aux relations d'emploi la rendent aujourd'hui beaucoup plus radicale. Il s'agit d'aller chercher dans des endroits où ils sont absents des

(16) $C f$. par exemple l'article pionnier de Jacques MAgaud (1974). salariés qui présentent des caractéristiques sociales éloignées de celles de la majorité de leurs mandants [...] L'enjeu est de savoir si le syndicalisme peut servir de lieu d'identification à une palette plus large de populations salariées, souvent en rupture avec les systèmes d'alliance internalisés au sein du salariat ancien» (Dufour, Hege, 2005, p. 21-22).

Cette dernière difficulté est donc d'autant plus épineuse que les «anciennes» réponses semblent encore les plus efficaces, comme on le voit avec le cas de La Poste. Ailleurs, c'est bien la capacité de l'ancienne forme syndicale à se redéployer sur d'autres bases, à la fois interprofessionnelles et territoriales, qui est en cause. Or, par exemple, «la conception interprofessionnelle de la vie syndicale est encore aujourd'hui dominée dans la CGT par l'idée que s'en font les statutaires, ce qui ne facilite guère la conquête de nouvelles terres de mission"» (PERNot, 2005, p. 188).

Opposer aux précarités professionnelles la perspective d'une «sécurisation sociale et professionnelle» est sans doute pertinent. À condition pour les syndicats de parvenir à la décliner concrètement à partir de luttes et d'expériences encore très embryonnaires, et de prendre en compte les perspectives - objectives comme subjectives, crédibles comme désirables - de sécurisation, du point de vue des salariés précaires concernés. On a peutêtre ici la meilleure clef de lecture des difficultés et des potentialités de l'action collective et syndicale sur nos trois terrains. La notion de sécurisation ne saurait prendre en effet le même visage à La Poste, sur les sites industriels aux collectivités de travail éclatées, et dans la restauration rapide. Il n'y a que dans le premier cas que la promotion vers le statut professionnel d'un noyau salarial central fasse encore sens à la fois dans les espérances individuelles des précaires et dans une stratégie collective de sécurisation. Dans le second cas l'unification du statut reste à re-construire sur des bases productives et territoriales incertaines. Dans le troisième l'amélioration substantielle de la condition salariale apparait comme un objectif hors de portée à court-terme, même si toute expérience laborieuse est porteuse d'apprentissages, - éventuellement enrichis par des pratiques de résistance ou d' action collectives - valorisables ultérieurement dans d'autres mondes professionnels. Dans le monde de la sous-traitance comme de la restauration rapide, obtenir effectivité du droit du travail et des relations professionnelles reste encore souvent le seul horizon d'une action syndicale sans cesse à reprendre.(17)

(17) On en trouvera des exemples dans la monographie réalisée par Jean-Marie Pernot sur l'expérience de L'Union syndicale multiprofessionnelle CGT du site des chantiers navals de SaintNazaire (PERnot, 2007). 
Redéployer vers le précariat les forces syndicales encore très largement ancrées dans le noyau protégé du salariat ne va pas sans fortes tensions. De nos trois terrains, seule la sous-traitance pétrochimique a été le lieu d'ébauches revendicatives allant dans ce sens. En même temps la résistance à la précarité dans les nouvelles générations, manifeste dans la restauration rapide comme dans d'autres luttes, est prometteuse. Une meilleure connaissance par les jeunes de leurs droits à l'orée de leurs premières expériences professionnelles dans ces vastes zones du précariat sans droit ne pourrait-elle pas être une des priorité de l'action syndicale?

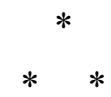

Bien d'autres défis sont posés au syndicalisme, entrevus sans être approfondis au cours de notre enquête, et qui participent de ses difficultés multiformes, y compris sur le terrain des précarités professionnelles: promouvoir en son sein des leaders représentatifs de la «diversité» actuelle du salariat précaire; sortir d'une relation de «don/contre don» aux salariés pour accompagner leur développement d'un «pouvoir d'agir» autonome (DAVEZIES, 2006); faire face à la disparition des grandes espérances sociales qui soutenaient l'action quotidienne, et des modes antérieurs d'agrégation de la représentation salariale (PERNOT, 2005) - dit autrement, prendre en comptel'enchâssement de la crise syndicale dans celle de la représentation politique; peser sur les orientations gestionnaires et stratégiques des firmes; nouer des alliances avec la nébuleuse d'autres mouvements sociaux contestant à leur manière le capitalisme libéral. Autant d'objets de recherches à développer.

\section{Bibliographie}

Amossé T. (2004), «Mythes et réalités de la syndicalisation en France», Premières synthèses, $n^{\circ} 44.2$, octobre.

Amossé T., Pignoni M.-T. (2006), «La transformation du paysage syndical depuis $1945 »$, Données sociales, Paris, Insee.

Amossé T., Chardon O. (2006), «Les travailleurs non qualifiés: une nouvelle classe?», Économie et statistique, $\mathrm{n}^{\circ} 393-394$.

Andolfatto D., LabBé D. (2006), «La transformation des syndicats français. Vers un nouveau modèle social?» Revue française de sciences politiques, vol. 56., $\mathrm{n}^{\circ} 2$.

BARNIER L.-M. (2006), «Du travail collectif au statut collectif» in Artous A. (eds), Travail critique du travail et émancipation, Paris, Syllepse.

Bouffartigue P., Pendariès J.-R., Peroumal F., PerRIN E. (Avec la participation de Bouteiller J., Contrepois S., Fribourg B., Jean R., Orban E., Samzun T.) (2007), Action collective et précarités. Le syndicalisme à l'épreuve. Enquête sur trois secteurs d'activité: Poste, sous-traitance pétrochimique, restauration rapide, LEST, Mars.

Bourhis A., Wills, T. (2001), «L'éclatement de l'emploi traditionnel. Les défis posés par la diversité des emplois typiques et atypiques », Relations industrielles, 56-1 : 5689.

Carlier A., Tenret E. (2007), «Des conflits du travail plus nombreux et plus diversifiés », Premières synthèses, 08-1.

Cartron D. (2005), «Engagement dans le travail et dans la grève chez Mac Donald's ", in Denis J.-M (eds), Le conflit en grève? Tendances et perspectives de la conflictualité contemporaine, Paris, La Dispute.

Cingolani, P. (2005), La Précarité, collection «Que-saisje», Paris, PUF.
DAVEZIES P. (2006), «Activité, subjectivité, santé», in Thery L. (eds), Le travail intenable, Paris, La Découverte.

Duffour C., Hege A. (2005), «Emplois précaires, emploi normal et syndicalisme», Chronique internationale de l'Ire, 97, pp. 5-21.

Mabrouki A. (2004), Génération précaire, Paris, Le cherche midi.

Magaud. J. (1974), «Vrais et faux salariés », Sociologie du travail, $\mathrm{n}^{\circ} 1$.

Martucelli D. (2004), "Figures de la domination», Revue française de sociologie, 45-3, pp. 469-497.

Mouriaux R. (2005), Le syndicalisme en France, collection «Que-sais-je», PUF.

Nkouachet R.-N. (2005), «La précarité de l'emploi au service de la prospérité du fast-food $»$, Sociologie du travail, $\mathrm{n}^{\circ} 4$.

Paugam, S. (2002), Le salarié de la précarité, collection «Quadrige», Paris, PUF.

Pernot, J.-M. (2005), Syndicats. Lendemains de crise?, collection «Folio Actuel», Paris, Gallimard.

Pernot, J.-M. (2007), Syndicats et sous-traitance aux Chantiers de l'Atlantique, IRES.

SEgRestin D. (1979), «Les communautés pertinentes de l'action collective», Revue française de sociologie, 29, 171-203.

ZARIFIAN P. (2003), La cohabitation fonctionnaires et agents contractuels à La Poste. État des lieux et perspectives, Mission de la recherche de La Poste.

ZARIFIAN P. (2005), «Tensions et esprit de révolte dans les entreprises de services », in Denis J.-M. (eds), Le conflit en grève? Tendances et perspectives de la conflictualité contemporaine, Paris, La Dispute. 